\title{
Extensión universitaria como vía para la prevención de la salud, municipio de Remedios, Cuba
}

\author{
University extension as a way for health prevention, Remedios municipality, Cuba
}

Luis Marcelino Correa Martin ${ }^{1}$

Blanca Ramona Tur García ${ }^{2}$

\section{Resumen}

Este trabajo ha mostrado la experiencia en la aplicación de acciones de la Extensión Universitaria, coordinadas por el Centro Universitario Municipal (cUM) y la Universidad Médica del municipio de Remedios, Cuba; se ha contribuido al trabajo preventivo de enfermedades para mejorar la calidad de vida de los pobladores de estas comunidades, municipio Monumento Nacional. Fue aplicada la investigación cuantitativa a través de las técnicas de entrevistas, encuestas, la observación y el procesamiento estadístico en una población de 24.000 habitantes, la muestra intencional no probabilística es de 56 familias que representan el $28.57 \%$ de la población que forma la comunidad rural de la zona, que es de 196 familias. El trabajo ha revelado resultados en cuanto al cuidado del medio ambiente, trabajo preventivo comunitario y elevación de la calidad de vida de los pobladores de estas comunidades.

Palabras clave: acción, enfermedades, extensión, prevención.

\section{Abstract}

This work has shown the experience in the application of actions of the University Extension, coordinated by the Municipal University Center (CUM) and the Medical University of Remedios municipality, Cuba; it has contributed to the preventive work of diseases, to improve life quality of the inhabitants of these communities, municipality National Monument. The quantitative research was applied through the techniques of interviews, surveys, observation and statistical processing in a population of 24,000 inhabitants, the non-probabilistic intentional sample is 56 families that represent $28.57 \%$ of the population that forms the rural community of the area, whose population is 196 families. The work has revealed results in terms of environmental care, community preventive work and improvement of the quality of life of the inhabitants of these communities.

Keywords: action, diseases, extension, prevention.

1 Máster en Ciencias, profesor Auxiliar de la UCLV, Universidad Central “Marta Abreu” de Las Villas. Correo: lcorrea@uclv.cu, ORCID https://orcid.org/oooo-0003-1093-3544

2 Máster en Ciencias de la Educación, profesora Auxiliar de la Universidad de Ciencias Médicas "Serafín Ruiz de Zárate Ruiz" de Villa

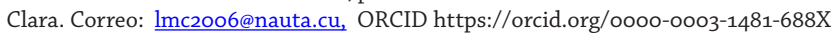

Recibido: 08/02/2021 - Aprobado: 25/11/2021 


\section{CIENCIAS SOCIALES}

\section{Introducción}

La actividad extensionista en las universidades juega un papel importante en la formación del profesional. Los estudiantes de la Universidad de Inglaterra, en el siglo XVIII, impartieron conocimientos teóricos para que los obreros pudieran asimilar los avances de la Ciencia y la Técnica en 1818, por primera vez en América Latina se habla de Extensión Universitaria como función social y fue en 1957 cuando se celebra la Primera Conferencia Latinoamericana de Extensión Universitaria, definiendo el concepto vinculado con la sociedad.

Mientras que, en Cuba (1922), la Reforma Universitaria dirigida por Julio Antonio Mella, crea la FEU y la Universidad Popular José Martí, la actividad extensionista con los altos estudios universitarios para obreros y campesinos, jugó un papel fundamental porque los proyectos de extensión universitaria vincularon a la universidad con los problemas de la comunidad tanto cultural, de salud, como económicos, para buscarle solución con rigor científico y académico.

Por esta tradición se desarrolla la actividad extensionista del Centro Universitario Municipal de Remedios con la Universidad Médica del municipio, vinculando el conocimiento científico y el trabajo preventivo con la población, con el objetivo de mejorar la calidad de vida de las familias en estas comunidades y realizando actividades preventivas para evitar enfermedades; trabajo extensionista que se desarrolló durante el 2019-2020, el cual logró transformaciones en el contexto social.

El Centro Universitario Municipal de Remedios aporta en estas acciones el nivel científico, psicológico y el adecuado conocimiento sobre la comunicación social y la investigación, mientras que la Universidad Médica aporta el conocimiento médico unido a las habilidades prácticas para realizar el trabajo preventivo desde la familia y el hogar en la comunidad, con la participación de los trabajadores y estudiantes que se vinculan a la Extensión Universitaria, acciones que se realizan de conjunto vinculando a la familia y la sociedad en general para lograr el objetivo propuesto.

El trabajo se realizó en Remedios, en tres Consejos Populares del municipio, donde la investigación arroja problemas de falta de higienización en la comunidad, pocas medidas preventivas en el consumo del agua, supuestamente potable, se detectaron problemas de contaminación, incorrecta cocción de alimentos de consumo diario, lo que provoca enfermedades diarreicas agudas, posibilidad de enfermedades tales como el dengue, el cólera, la hepatitis y otras que, por la falta de conocimiento, la población no toma las medidas de carácter preventivo, a pesar de los esfuerzos que hace la salud pública.

Las familias que se consideran con factores de riesgo en la comunidad disminuyeron de un $44.28 \%$ a un $4.92 \%$, por lo que mejoró la calidad de vida de la población en 
el contexto comunitario, atenuando las enfermedades prevenibles y propiciando un mejor estado de salud donde se promueven las acciones extensionistas, lo que implica propiciar una mejor calidad de vida para niños, jóvenes y adultos.

Las acciones extensionistas realizadas con la participación del Centro Universitario Municipal, en coordinación con la Universidad de Ciencias Médicas del territorio, tuvieron un impacto social que transformó los modos de actuación de los pobladores y mejoró la comunicación social entre los habitantes con transformaciones evidentes en cuanto a la higienización, consumo de alimentos no aptos para la vida y la prevención de enfermedades.

\section{Materiales y métodos}

Es una investigación cuantitativa donde se realizó el procesamiento estadístico en una población de 24.000 habitantes, la muestra intencional no probabilística es de 56 familias que representan el $28.57 \%$ de la población que forma la comunidad rural de la zona, que es de 196 familias.

Las técnicas aplicadas fueron la encuesta, la entrevista, el debate, la reflexión, la terapia ocupacional, entre otras, que dieron la posibilidad de un trabajo preventivo a partir del conocimiento teórico y práctico de la problemática tratada; en conjunto con los métodos utilizados en la realización de este trabajo, se utilizó pancartas, diapositivas, entre otros recursos que posibilitó la aplicación de las técnicas participativas en el contexto comunitario y social.

- La encuesta: permitió determinar los problemas existentes en cuanto al conocimiento de las enfermedades prevenibles y cómo evitarlas.

- La entrevista: permitió corroborar la situación familiar en cuanto al cumplimiento de las medidas de carácter preventivo que puede realizar la población en la comunidad y el contexto familiar.

- La observación: brindó la posibilidad de determinar las causas de las enfermedades en el contexto familiar y comunitario.

- El matemático y/o procesamiento estadístico: permitió hacer las tabulaciones necesarias de los resultados y utilizar la estadística descriptiva para hacer explicaciones lógicas del problema. 


\section{Fundamentación de la propuesta}

La universalización de la Educación Superior en Cuba es una realidad, se justifica la necesidad de una estrategia que fortalezca, desde la promoción de salud, el vínculo Universidad-Sociedad y el trabajo preventivo con carácter extensionista, así lo expresó González Fernández L., y González G. (2003): "La extensión universitaria es el proceso universitario que tiene como propósito promover cultura en la comunidad interuniversitaria y extrauniversitaria".

Es la comunidad extrauniversitaria donde se aplicó las acciones que incidieron en el contexto familiar, que integra la comunidad de Remedios, incidiendo de esta forma en la población en general que convive en estas comunidades intrincadas del territorio local.

Asimismo, Gutiérrez R. (2002) manifestó que la extensión universitaria “(...) responde a la necesidad social de preservar, desarrollar y promover la cultura en la comunidad interuniversitaria y extrauniversitaria para contribuir a su desarrollo" (p.2).

Las acciones se diseñaron de forma tal que promovieran la cultura en las comunidades y contribuyó a un desarrollo higiénico sanitario que permitió prevenir enfermedades y mejorar la calidad de vida de sus pobladores. Por tanto, la razón de ser de la universidad contemporánea es satisfacer las necesidades sociales e individuales, a partir de la preparación del ser humano como ente activo en la sociedad en la que se desempeña. Para poder dar cumplimiento a este cometido, la universidad, en tanto, institución formativa y transformadora, requiere aglutinar todo su quehacer y proyectarlo a través de un proceso fundamental: la Extensión Universitaria.

La Extensión Universitaria, como vía para la realización del trabajo preventivo en la comunidad, posibilitó el desarrollo de acciones extensionistas cumpliendo con las siguientes funciones:

1. Función educativa: cuando se trabaja la educación higiénico sanitaria en el contexto comunitario donde inciden las acciones.

2. Función preventiva: contribuyó a prevenir enfermedades en niños, jóvenes y en el adulto mayor, causadas por la falta de higiene y el consumo de alimentos sin la adecuada elaboración.

3. Función investigativa: contribuyó a desarrollar la labor investigativa de los estudiantes en el terreno comunitario, determinando las causas de enfermedades tales como diarrea, hepatitis, dengue, cólera. 
4. Función ambientalista: favoreció el saneamiento de la comunidad, eliminando microvertederos y vectores que pueden ocasionar enfermedades prevenibles.

5. Función gerencial: cuando se empleó capacidades y conocimiento de la medicina por los estudiantes y docentes del Centro Universitario Municipal, para dirigir, controlar y valorar los resultados de la actividad extensionista realizada.

Los resultados en las acciones de carácter extensionista se vincularon a los programas de salud a nivel de país. Las acciones se proyectaron, desde el inicio, con un carácter educativo, tomando el criterio de Demerval y Cortez (1997) que plantearon que la educación es un fenómeno histórico y social, responsable de la transmisión de la cultura y de la acción mediadora entre lo cotidiano y lo no cotidiano en la formación del individuo, por lo que se trabajó en la comunidad, en función de hacer entender la importancia de la labor preventiva y poder aplicar una atención integral por parte de los estudiantes, en la actividad extensionista comunitaria, donde los estudiantes y docentes, trasmitieron conocimientos a la población así como trabajo preventivo, higiénico sanitario y medioambientalista para contribuir a mejorar la calidad de vida de la población, tomando en cuenta el criterio de Pablo Freire (1973) cuando dijo:

Comencemos por afirmar que solamente el hombre, como un ser que trabaja, que tiene un pensamiento-lenguaje, que actúa, y es capaz de reflexionar, sobre sí mismo, y sobre su propia actividad, al alcanzar tales niveles es un ser de praxis. Un ser de relaciones en un mundo de relaciones, las acciones extensionistas se diseñaron teniendo en cuenta que fueran dirigidas a que el hombre piense y con su propio lenguaje sea capaz de analizar y trasmitir en la práctica diaria con sus relaciones, la necesidad de cumplir por los demás las medidas establecidas para prevenir el contagio de la pandemia. (p.49)

\section{Propuestas de acciones de Extensión Universitaria}

\section{Acciones del Centro Universitario Municipal}

\section{Objetivos:}

1. Monitorear las coordinaciones con los directivos de los Consejos Populares e impartir los primeros conocimientos sobre temas que van a trabajar los estudiantes y profesores como acciones de Extensión Universitaria.

2. Coordinar con los presidentes de los Consejos populares para la realización de talleres y conferencias sobre el cuidado del medio ambiente y los riesgos de la contaminación ambiental en la comunidad. 


\section{CIENCIAS SOCIALES}

3. Impartir temas, conferencias y realizar talleres con directivos de las organizaciones de masas y familias líderes en la comunidad, donde se trabajen temas relacionados con el trabajo en equipo para lograr la higienización de las áreas designadas.

4. Desarrollar charlas instructivas en la comunidad y Consejos Populares sobre la higienización, su importancia y consecuencias para la salud y la mejor calidad de vida de la población.

5. Aplicar el Proyecto Comunitario "Mi familia y yo aportamos a la Comunidad" en los tres Consejos Populares.

\section{Acciones del Centro Universitario Municipal con estudiantes de la Universidad Médica}

1. Asesoramiento a los estudiantes en Metodología de la Investigación para propiciar la realización del diagnóstico inicial.

2. Adiestramiento en el tema relacionado con la Comunicación Social.

3. Asesoramiento en la elaboración de proyectos de investigación en los distintos Consejos Populares.

4. Asesoramiento en la elaboración de instrumentos investigativos de diagnóstico y tabulación de resultados aportados por la aplicación de estos.

5. Impartición de conferencias a los estudiantes sobre el tema de valores y ética profesional con énfasis en la responsabilidad, honestidad, laboriosidad y humanismo como futuros profesionales de la salud.

\section{Acciones Extensionistas realizadas por la Universidad Médica}

Objetivo: Ejecutar las acciones de Extensión Universitaria de forma práctica en la comunidad para lograr un mejor trabajo preventivo sobre la higienización, cuidado del medio ambiente y prevenir causas que pueden producir enfermedades no trasmisibles en niños, jóvenes y adultos que interfieren la calidad de vida.

\section{Acciones}

1. Visitas de atención médica a centros de salud donde se tienen internadas personas de la tercera edad (Asilos de Ancianos y Casas de abuelos) de la localidad, con estudiantes de cuarto año de Medicina. 
2. Desarrollo de charlas educativas sobre prevención de salud en zonas de la comunidad más vulnerables a enfermedades por mal elaboración de alimentos e inadecuada higienización.

3. Control y evaluación del trabajo comunitario realizado por los estudiantes como vía de Extensión Universitaria en los distintos Consejos Populares.

4. Desarrollo, en la comunidad, de la campaña de termometría en familias de los distintos Consejos Populares.

5. Desarrollo de la campaña antivectorial con carácter preventivo.

6. Inspecciones y visitas a familias diagnosticadas con riesgos de falta de higiene en cada Consejo Popular (hacer trabajo educativo y preventivo).

7. Coordinación y participación en campañas de saneamiento ambiental en el contexto comunitario.

8. Impartición de charlas educativas en los policlínicos más cercanos y áreas de salud de la comunidad.

9. Desarrollo de visitas de terreno a familias con posibles riesgos a contraer Dengue o Cólera.

10. Realización de conversatorios, charlas y conferencias sobre enfermedades prevenibles en el contexto familiar y comunitario.

11. Realización de visitas al hogar para hacer trabajo preventivo, con énfasis en la elaboración de alimentos y consumo adecuado del agua potable por niños y familia en general.

12. Visitas a centros educacionales para hacer trabajo preventivo sobre la importancia de la higiene personal y ambiental a través de charlas, conversatorios con estudiantes y docentes, conferencias y entrega de propagandas gráficas existentes.

13. Atención especializada, con carácter preventivo, a embarazadas y personas de la tercera edad. 


\section{CIENCIAS SOCIALES}

\section{Conclusiones}

El impacto social en la comunidad fue positivo, las familias afectadas con alta posibilidad de riesgos a adquirir enfermedades por inadecuado consumo de alimentos, falta de higiene personal y familiar, disminuyó de un $44.28 \%$ a un $4.92 \%$, en los tres Consejos Populares seleccionados: El Carmen, San Salvador y Hermanos Herradas; el trabajo preventivo fue efectivo con las acciones extensionistas de los estudiantes universitarios, se transformaron los modos de actuación de la población, a través de las actividades y acciones.

Se contribuyó a disminuir los microvertederos que favorecían la contaminación ambiental comunitaria y existencia de vectores, se eliminaron los estanques sin la debida protección y falta de abate establecido; se logró un consumo de agua hervida o con hipoclorito en el $90 \%$ de la población, se adquirió consciencia y educación sanitaria que implicó una mejor calidad de vida en el contexto comunitario de cada Consejo Popular. Se contribuyó con éxito a:

1. Mejorar la higiene colectiva, familiar e individual.

2. Se eliminaron microvertederos que provocan vectores causantes de enfermedades prevenibles.

3. Se mejoraron los modos de actuación de pobladores en cuanto al cuidado del medio ambiente y consumo de agua no potable.

4. Se contribuyó a mejorar la calidad de vida de los niños, jóvenes y adultos de estas comunidades.

5. Las actividades extensionistas sirvieron de apoyo al trabajo desarrollado por el Ministerio de Salud Pública en cuanto a la disminución de enfermedades prevenibles y la realización de un fuerte trabajo de divulgación de salud en cada territorio, por lo que se generalizó en todos los poblados del municipio de Remedios.

\section{Lecciones aprendidas}

1. Las familias que se consideran con factores de riesgo en la comunidad, disminuyeron de un $44.28 \%$ a un $4.92 \%$ por lo que mejoró la calidad de vida de la población en el contexto comunitario.

2. Las acciones extensionistas realizadas con la participación del Centro Universitario Municipal, en coordinación con la Universidad de Ciencias Médicas del territorio, tuvo un impacto social que transformó los modos de actuación de los pobladores 
y mejoró la comunicación social entre los habitantes, con transformaciones evidentes en cuanto a la higienización, consumo de alimentos no aptos para la vida y la prevención de enfermedades.

\section{Lista de referencias}

Demerval Saviann, S., y Cortez, R. (1997). Escuela y Democracia. Autores asociados, San Pablo. España.

Freire Paulo. (1973). ¿Extensión o Comunicación? Primera Edición. México. Editores S.A. C/Plaza 5. Madrid 33 España. p-49.

González Fernández L.M, y González G. (2003). Extensión Universitaria Principales tendencias en su evolución y desarrollo. Revista Cubana de Educación Superior No 39 La Habana. (2007). http//www/dictun cu//Revistas/EducaciónSuperior/12003/ Art/2002003pdf.

Gutiérrez Hernández, R. (2002). La Extensión Universitaria en los Institutos Superiores Pedagógicos en la Universalización. ISP "Felix Varela” Villa Clara. Cuba (p-6). 